\title{
Planteamiento de un modelo logístico para reducir costos del subproceso de pintura en muebles Bovel Ltda.
}

\author{
Logistic model approach to reduce costs in furniture painting thread Bovel Ltda.
}

\author{
Silvia Natalia Rave Arias, Diana Marcela Arias Acevedo, Jorge Mario García Osorio \\ Especialización en Logística Empresaria, Escuela de Tecnología Industrial, Universidad Tecnológica de Pereira, Pereira, \\ Colombia \\ Correo-e: sinara@utp.edu.co
}

\begin{abstract}
Resumen- Por medio de un análisis del subproceso de pintura en la fábrica Muebles Bovel Ltda. se plantea un sistema que permita minimizar los reprocesos y reducir los costos de los mismos, identificando el comportamiento de los costos presentes en ordenes de producción frente a los costos estándar definidos para el proceso, analizando el origen de los productos no conformes, $y$ permitiendo proponer modificaciones al proceso, brindando herramientas y técnicas para generar valor agregado a los productos donde los márgenes de rentabilidad sean cada vez más altos, minimizando el alto impacto que generan los reprocesos y optimizando los recursos.
\end{abstract}

Palabras clave - Calidad, sistemas de Costeo, Operaciones, Logistica.

\begin{abstract}
Through an analysis of the painting thread Bovel Furniture Factory Ltd arises a system to minimize and reduce rework costs thereof, identifying the behavior of these costs in production orders against defined standard costs for the process, analyzing the origin of non-compliant products, and allowing propose changes to the process, providing tools and techniques to add value to the products where profit margins are higher and higher, minimizing high impact generated by reprocessing and optimizing resource.
\end{abstract}

Key Word - Quality, Cost Study, study methods and timing, logistics..

\section{INTRODUCCIÓN}

Con mercados cada vez más exigentes, es importante que las organizaciones identifiquen los productos, servicios y necesidades que se acomodan más a las expectativas de los potenciales clientes, llevándolas a afrontar retos con miras a la expansión y perfeccionamiento de las técnicas propias de una operación. Las exigencias del medio y la alta competitividad, lleva al perfeccionamiento de las operaciones y a la búsqueda de la optimización de sus recursos, buscando ofrecer productos de alta calidad, con mayor eficiencia y productividad, a un menor costo. $\mathrm{Y}$ en esta medida, la exigencia al cambio es cada vez mayor, allí es donde aparece la logística como un elemento diferenciador que permite observar a la organización como un elemento integrador.

La logística ha sido vista durante décadas como la que orienta los procesos de abastecimientos, recepción, distribución y almacenamiento, sin ninguna relación con los demás procesos de la organización; pero la realidad es que la logística es el proceso integrador por excelencia en una empresa.

En este artículo, se plantea un proyecto con base en el análisis de la problemática en el proceso de pintura en Muebles Bovel Ltda, teniendo como objeto principal de estudio la disponibilidad y preservación de insumos, recursos y/o información, identificando y estableciendo las causas principales de productos defectuosos y los reprocesos en que incurre la organización, así como el impacto operacional y económico generado por los mismos, debido principalmente a fallas en el almacenamiento de producto en proceso.

De esta forma se plantea una alternativa que permita disminuir los reprocesos y los costos de producción; permitiendo mejorar la productividad y la calidad del producto, esto, mediante el análisis del flujo, de costos, de almacenamiento de producto en proceso y proponiendo un nuevo esquema de distribución en planta para Muebles Bovel Ltda.

\section{CONTENIDO}

\section{A. Identificando un problema}

Este artículo se basa en la experiencia producto del desarrollo de un proyecto realizado en la empresa Muebles Bovel Ltda, organización dedicada a la fabricación de muebles en madera línea hogar (alcobas, comedores, salas y accesorios), fundada en la década de los 80 por su presidente Darío Botero Gómez. 
En la empresa Muebles Bovel Ltda, se identifican las siguientes secciones en el área de producción: Premaquinado, Maquinado, Ensamble, Lijado, Tapicería, Empaque y Despachos, y Pintura (Aplicación de color, suavizado y aplicación de laca, secado e inspección), siendo esta última, el área más sensible de la fábrica y en la cual se basó el estudio realizado. Hacer que el producto fluya a lo largo de la línea es vital, sin embargo, en dicha sección se presentan fallas operativas ocasionando un alto porcentaje de productos defectuoso.

\section{B. Objetivo del proyecto}

Plantear un modelo logístico que ayude a reducir los reprocesos y minimizar los costos en el subproceso de pintura en la empresa Muebles Bovel Ltda., generando una mayor productividad y calidad.

\section{Recolección y análisis de datos}

\section{Causa y efecto}

Para tener mayor claridad de la problemática afrontada por la organización, se realizó un análisis de espina de pescado o diagrama de causa y efecto, ver Figura 1. del cual se obtuvo la siguiente información:

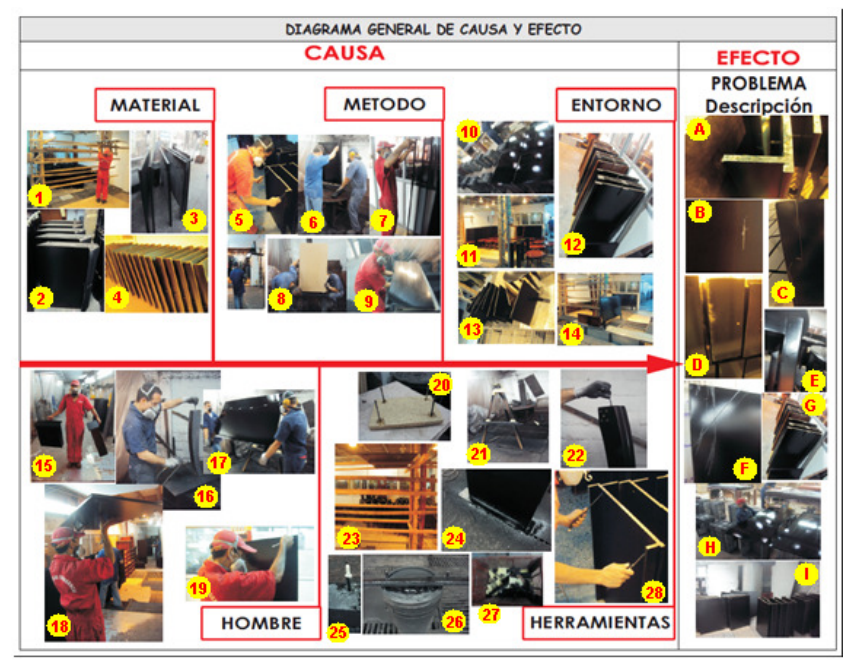

Figura 1. Diagrama causa y Efecto, Muebles Bovel Ltda.

\section{CAUSAS:}

- MATERIAL: Ubicación de elementos adicionales o temporales en infraestructura para optimizar el espacio en el área de secado. Se cuenta con un producto que es manejado en piezas durante toda la línea de producción. Piezas de gran tamaño en ancho y altura, almacenadas a piso (piso sin acabado rugoso y áspero). Ubicación de las piezas de manera vertical sostenidas de los laterales donde se pueda provocar un efecto dominó, por ser piezas pintadas hasta 6 caras.
MÉTODO: improvisación en el uso de herramientas para la manipulación de las piezas. Uso inadecuado de elementos del entorno no aptos para la sujeción, manipulación y transporte de las herramientas. Manipulación y transporte de piezas sobredimensionadas para el patinador, provocando daños en la persona. Falta de control en la segregación de funciones, varias personas ubican piezas antes de ser pintadas. Piezas que deben ser pintadas por varias caras, requiriendo ser pintadas por intervalos.

- ENTORNO: Almacenamiento para secado de las piezas sobre canastas. Piso de cabina de secado elevado por inyectores y eyectores. Ubicación de las piezas para ser secadas en la cabina de pintura de manera vertical sin ningún tipo de separación para evitar el efecto dominó. Piezas que por su tamaño deben ser ubicadas de manera vertical, pero para lograrlo se deben adicionar piezas de madera y ser fijadas a la pieza con puntillas, lo que provoca deterioros y fractura del producto. Escalón de $30 \mathrm{~cm}$ en la cabina de pintura, dificultando la manipulación y transporte de piezas pintadas.

HOMBRE: Manipulación de las piezas pintadas una a una, corriendo el riesgo de roses y presentar pintura corrida o defectuosa. Uso inadecuado de elementos de sujeción para facilitar la fijación de las piezas y permitir la aplicación de pintura a varias caras. Falta de control y estandarización del proceso productivo, cada pintor tiene su forma de trabajo. Para material sobredimensionado, se requiera más de una persona para la manipulación y transporte de piezas. Levantamiento de cargas por encima de los hombros. Uso de herramientas primarias.

- HERRAMIENTAS: Uso de clavos o puntillas para la fijación de piezas y evitar que se caigan durante el proceso de pintura y secado. Uso de trípodes o bancos para pintar las piezas, los cuales limitan e número de caras a ser pintadas. Uso de punzones para la manipulación de las piezas y giros de las mismas. Uso de listones en madera para el secado horizontal de las piezas. Utilización de piezas de madera con pines o salientes que permitan fijar las piezas largas. Uso de Baldes plásticos con el fin de aislar las piezas del suelo y facilitar la manipulación de la misma al tenerla a la altura adecuada. Uso de retales de madera, los cuales después de mucha manipulación pueden quebrarse durante el desarrollo de la actividad y dejar caer la pieza que sostiene.

\section{EFECTOS:}

- Cantos de las piezas con suciedades y pegotes de pintura lo que provoca que finalmente las piezas no encajen o se tenga una actividad adicional de retirar las rebabas de pintura antes de ser empacadas.

- Rayones de las piezas por el uso de punzones para la manipulación y transporte de piezas ya pintadas.

- $\quad$ Rayones de piezas por caídas 
- Suciedad en el momento de la aplicación de la pintura, bancos de pintura con residuos y caídas del arrume.

- Goteos por aplicación de pintura provocados por exceso de pintura o técnica del pintor

- Rayones de las piezas provocados por caídas según efecto dominó.

- Almacenamiento de piezas en posición vertical sostenidas de la pared y a piso

- Secado de piezas en sellador sobre elementos no aptos como canastas y canecas

- Piezas que después de ser pintadas deben ser dejadas cerca a las cabinas pintura por que el patinador no llega a tiempo para el traslado de las mismas o que por falta de espacio deben ser ubicadas allí, o que les falte partes para ser pintadas.

\section{Recolección de información}

Se hace una clasificación de las piezas, buscando similitudes entre las mismas:

- Clasificación 1. Costado y Espaldar en el Closet Moderno; Espaldar izquierdo, Nave grande y Costado intermedio en el Multimueble.

- Clasificación 2. Entrepaño, División Alta y Nave en el Closet Moderno; Costado, División Baja, Cubierta, Nave pequeña y Nave derecha en el Multimueble; Costado, Cubierta y Espaldares / Costados en el Centro Entretenimiento.

- Clasificación 3. División Baja en el Closet Moderno y Piso/base en el Multimueble.

- Clasificación 4. Entrepaño móvil del Closet Moderno y la Cubierta pequeña del Multimueble.

- Clasificación 5. Gavetas del Closet Moderno, Multimueble o Centro Entretenimiento.

- Clasificación 6. División Alta y Refuerzo espaldar en el Multimueble.

Se establecen los productos no conformes (PNC), según las causas anteriormente establecidas y ya analizadas, para cada tipo de clasificación, ver Tabla 1.

\begin{tabular}{|l|l|l|}
\hline $\begin{array}{l}\text { SIMILITUD } \\
\text { TAMAÑO }\end{array}$ & $\begin{array}{l}\text { CANTIDAD } \\
\text { (PNC) }\end{array}$ & $\%$ \\
\hline 1 & 73 & $10,49 \%$ \\
\hline 2 & 299 & $42,96 \%$ \\
\hline 3 & 12 & $1,72 \%$ \\
\hline 4 & 131 & $18,82 \%$ \\
\hline 5 & 167 & $23,99 \%$ \\
\hline 6 & 14 & $2,01 \%$ \\
\hline TOTAL & 696 & $100,00 \%$ \\
\hline
\end{tabular}

Tabla 1. Cantidad de reprocesos agrupados por tamaño de las piezas

Las piezas donde más se presentan reprocesos son las que se clasifican como 2 con un 43,08\%
Para profundizar en la problemática ya establecida, se realiza estudio de tiempos y movimientos en el proceso de pintura, y se establecen los tiempos para cada tipo de pieza

Se establece el comportamiento de producto no conforme dentro del proceso de pintura, ver Tabla 2 .

\begin{tabular}{|c|c|c|c|}
\hline TIPO DE ACTIVIDAD & CAUSA & CANTIDAD & PORCENTAJE \\
\hline \multirow{4}{*}{ MOVIMIENTOS } & $\begin{array}{l}\text { Superficie } \\
\text { rayada }\end{array}$ & 57 & $8,19 \%$ \\
\hline & Golpes & 85 & $12,21 \%$ \\
\hline & Manipulación & 53 & $7,61 \%$ \\
\hline & $\begin{array}{l}\text { Arrume } \\
\text { defectuoso }\end{array}$ & 104 & $14,94 \%$ \\
\hline \multirow{4}{*}{ TÉCNICA } & $\begin{array}{l}\text { Superficie } \\
\text { sucia }\end{array}$ & 112 & $16,09 \%$ \\
\hline & Goteado & 63 & $9,05 \%$ \\
\hline & Falta color & 78 & $11,21 \%$ \\
\hline & $\begin{array}{l}\text { Color } \\
\text { incorrecto }\end{array}$ & 20 & $2,87 \%$ \\
\hline \multirow{4}{*}{ OTRA } & Porosidad & 80 & $11,49 \%$ \\
\hline & Detallado & 10 & $1,44 \%$ \\
\hline & $\begin{array}{l}\text { Maquinado } \\
\text { errado }\end{array}$ & 4 & $0,57 \%$ \\
\hline & $\begin{array}{l}\text { Adición } \\
\text { piezas }\end{array}$ & 30 & $4,31 \%$ \\
\hline \multicolumn{2}{|l|}{ TOTAL } & 696 & $100,00 \%$ \\
\hline
\end{tabular}

Tabla 2. Comportamiento de causas de producto no conforme

\section{Análisis de información}

Del análisis de tiempos y movimientos en el proceso de pintura, se encuentra que el transporte de materiales es muy representativo dentro de este. Ver Figura 2.

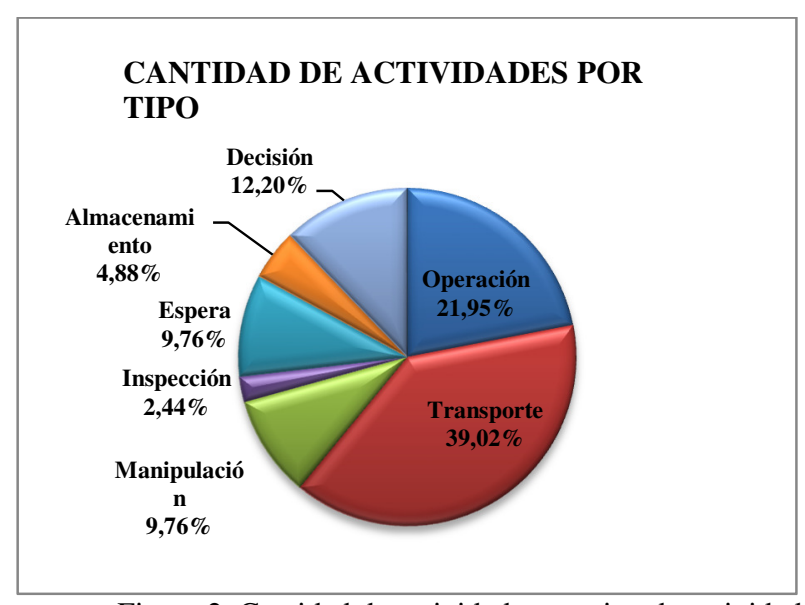

Figura 2. Cantidad de actividades por tipo de actividad. 
Del análisis de tiempos y movimientos, para cada tipo de pieza, se obtuvo un resultado similar, ver Figura 3. El comportamiento de las gráficas para cada tipo de pieza en el subproceso de pintura de Muebles Bovel Ltda. Fue similar. Aunque se encontraron unas pequeñas variaciones, se pudo identificar claramente que en todos los casos las actividades que demandan más tiempo son las esperas y los almacenamientos, teniendo en cuenta que dentro del cálculo de estos tiempos no se consideró el tiempo de almacenamiento en la cabina de secado, por ser un tiempo atípico (de $1 / 2$ hora hasta 24 horas), cuando los tiempos totales de las otras actividades máximo son de 2 horas.

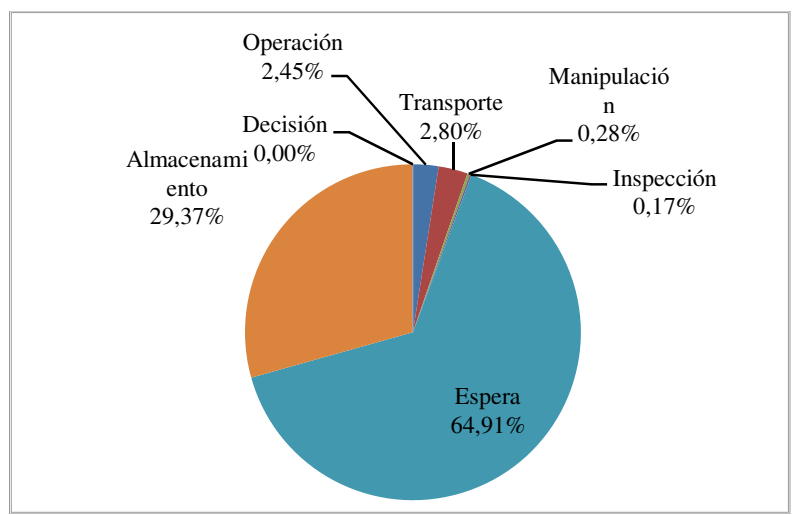

Figura 3. Tiempo total por tipo de actividad (cubierta fija).

Del análisis de producto no conforme, identificando el comportamiento de cada una de las causas, ver figura 4., se pudo determinar que el $42,96 \%$ del Producto No Conforme se debe a causas relacionadas con los movimientos o manipulaciones de los que son objeto las piezas en el subproceso de pintura.

De este $42,96 \%$, la mayor cantidad, un $14,94 \%$ se debe a arrume defectuoso, que es la forma como se denominan a los daños producidos por mala acomodación de las piezas, ya sea por contacto entre ellas o con otras superficies.

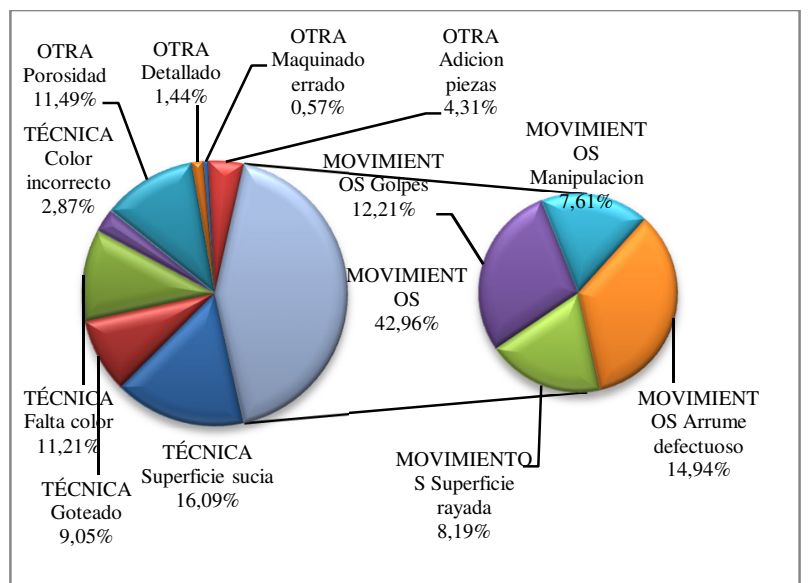

Figura 4. Causas del producto no conforme (movimientos).

\section{Análisis de costos}

5.

Para el análisis de los costos del producto, se establece:

Costo estándar por órdenes de producción, para cada tipo de mueble. Costo estándar por subprocesos. Costo por orden de producción. Costo de producto no conforme por órdenes de producción, ver figura 5.Costo de producto no conforme por piezas. Y finalmente se analiza la incidencia de los costos en la rentabilidad del negocio, ver tabla.

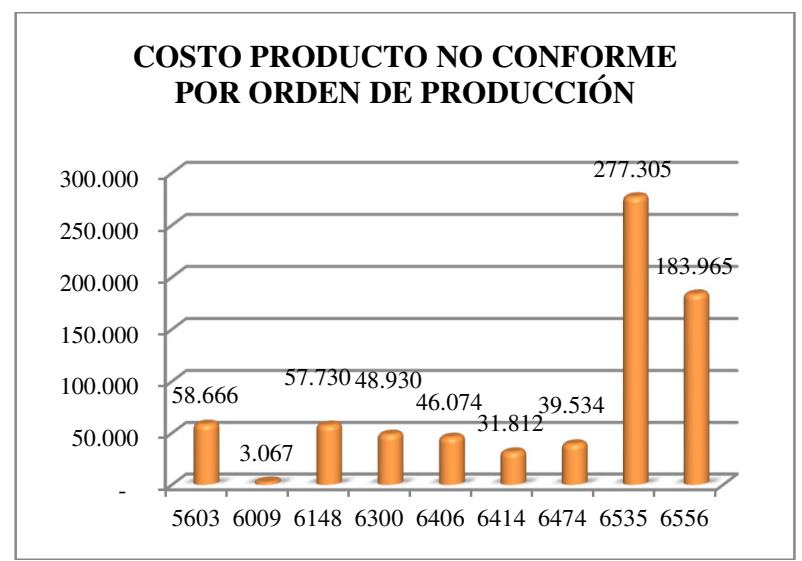

Figura 5. Comportamiento costo producto no conforme

Todos los costos de la no calidad serán asumidos en su totalidad por la fábrica, encontrando que en algunas ocasiones dicha rentabilidad es negativa, adicional a ello, se debe tener en cuenta que el presente análisis excluye los costos indirectos de fabricación, los cuales no están definidos en el modelo de costos de la empresa.

En este orden de ideas, se puede apreciar que la utilidad de la empresa se reduce su mínima expresión, pudiendo afectar, la rentabilidad de esta.

Al analizar el comportamiento de los costos por ordenes de producción, tomando varias de estas y analizando su información, se puede determinar que la variación frente al costo estándar es negativa, esto se debe a la cantidad de producto defectuosos y los reprocesos incurridos a causa de estos. Ver Tabla 3.

\begin{tabular}{|l|c|l|}
\hline Orden producción & Costo op & $\begin{array}{l}\text { Variación } \\
\text { respecto al op } \\
\text { estándar }\end{array}$ \\
\hline 6269 & 423.730 & $-66,04 \%$ \\
\hline 6342 & 311.780 & $-22,17 \%$ \\
\hline 6414 & 349.061 & $-36,78 \%$ \\
\hline 6535 & 327.546 & $-28,35 \%$ \\
\hline 5980 & 257.957 & $-1,08 \%$ \\
\hline 6009 & 239.563 & $6,13 \%$ \\
\hline 6148 & 601.469 & $-135,68 \%$ \\
\hline Costo estándar & 255.201 & \multicolumn{2}{|l}{} \\
\hline
\end{tabular}

Tabla 3. Variación Orden de producción Vs. Costo estándar 


\section{PROPUESTA}

Como desarrollo de la propuesta buscando minimizar los errores en el proceso por las causas identificadas, se plantea nuevas herramientas y redistribución en área de trabajo, así:

\section{Herramientas a implementar:}

- Carro con rodamientos, ideal para el transporte de piezas pequeñas. Ver figura 6. Facilitando el almacenamiento temporal y desplazamiento adecuado de piezas, evitando desplazamiento de piezas individualmente, reduciendo el número de desplazamiento por operario.

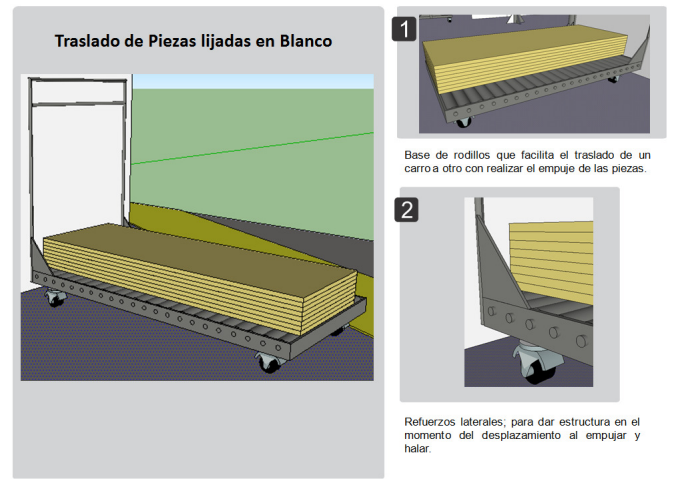

Figura 6. Carro con rodachinas

- Sujetador de pizas, sistema que permite optimización en el espacio de almacenamiento y facilita la manipulación y secado de las mismas. Reduciendo la manipulación de las piezas y por tal razón, se reduce la cantidad de producto defectuoso por técnica o manipulación, ver Figura 7.
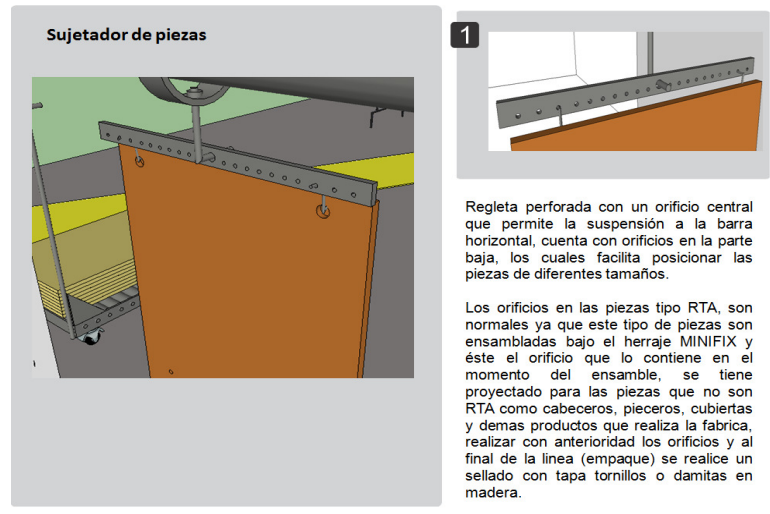

Figura 7. Sujetador de piezas

Sistema de aplicación de sellado, con sujetadores que permiten facilitar el almacenamiento y manipulación de piezas. Reduciendo el contacto con el producto durante el proceso de pintura, facilitando la realización del proceso y minimizando reprocesos. Ver figura 8.

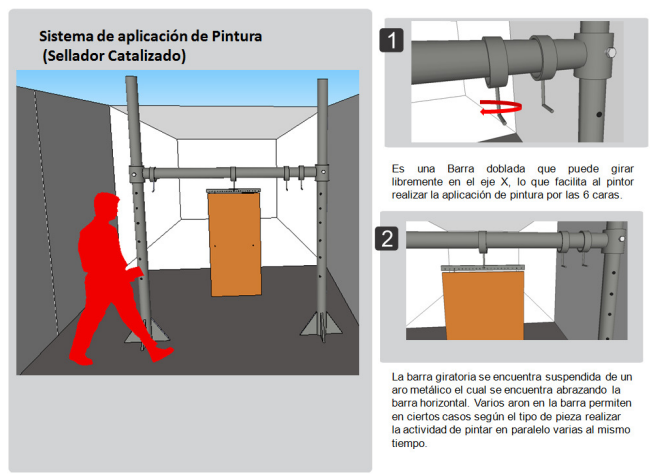

Figura 8. Sistema de Aplicación de Pintura

- Sistemas de rieles en techo. Permiten el desplazamiento adecuado de material en proceso, facilita almacenamientos y operación sobre la pieza. Ver figura 9 .

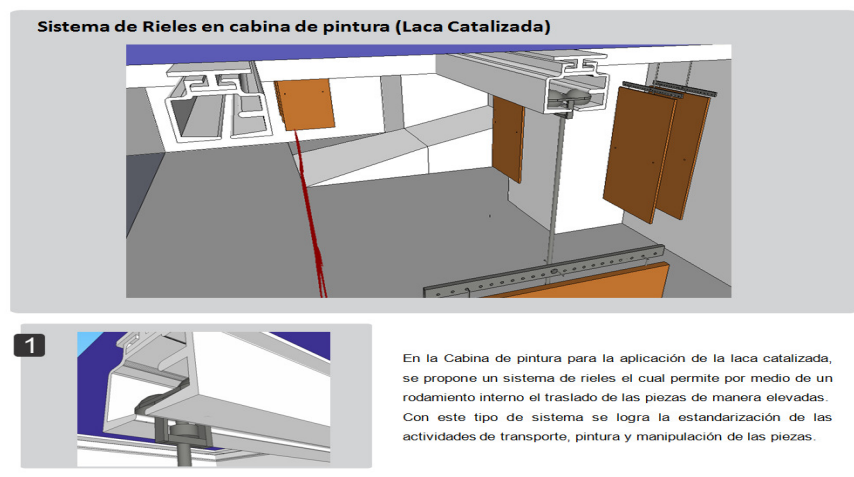

Figura 9. Sistema de Rieles

- Zona de almacenamiento. Proporciona el espacio suficiente y adecuado para el almacenamiento, minimizando el riesgo de dañar las piezas. Ver figura 10.

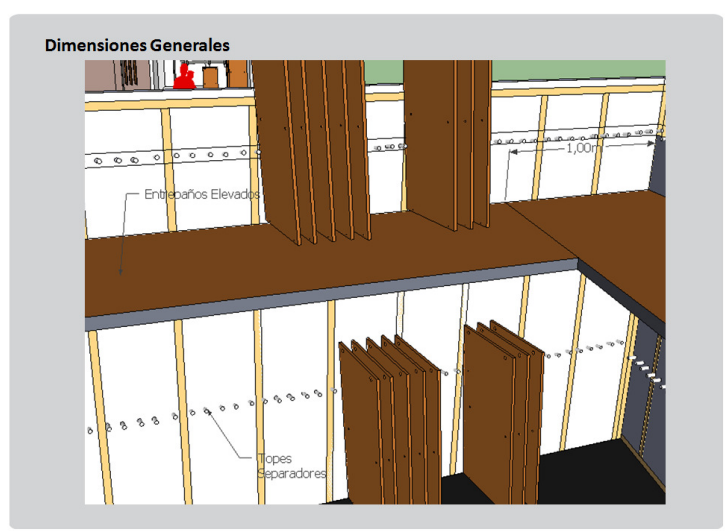

Figura 10. Bodega o zona de almacenamiento

2. Distribución de planta

Para facilitar la operación, minimizando desplazamientos, improvisación de operaciones y facilitando los desplazamientos con las piezas, se plantea un modelo de distribución en planta implementando las herramientas antes mencionadas, estos se 
pueden apreciar en las figura 11, visualizando el modelo actual y el propuesto.

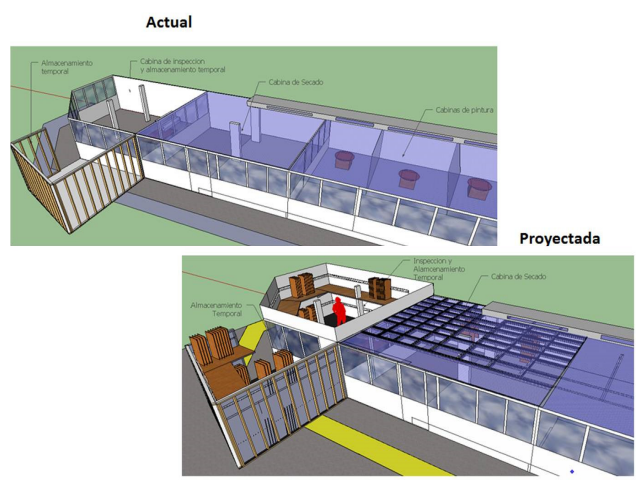

Figura 11. Distribución en planta

\section{ANALISIS ACTIVIDADES CON LA PROPUESTA}

Finalmente, para probar la eficiencia en el modelo logístico planteado, se analiza mediante estudio de tiempos y movimientos estableciendo las nuevas actividades a desarrollar con los tiempos estimados de cada una de estas. Los resultados se muestran a continuación, ver Tabla 4.

Encontrando las siguientes mejoras:

- Disminución en el número total de actividades de 41 actividades que se tenían identificadas se llegó a 29.

- Las operaciones se disminuirían por el hecho de que las actividades de pintura para las múltiples caras se realizan de una vez tanto para el sellador catalizado, como para la laca catalizada.

- Los transportes se reducirían a la mitad en número, y se espera una reducción en tiempo, puesto que se contará con ayudas mecánicas, además de permitir el desplazamiento de varias piezas simultáneamente.

- Las manipulaciones de las piezas tuvieron un incremento, pero ya se trata de actividades de cargue y descargue de las piezas en los equipos de transporte diseñados.

- La inspección se mantiene como actividad, puesto que es indispensable asegurar productos de óptima calidad, al igual que los almacenamientos en las bodegas temporales

\section{CONCLUSIONES}

Las variables que más impactan y afectan la calidad y productividad en el subproceso de pinturas en Muebles Bovel, son: reprocesos por mala manipulación o almacenamiento y las ineficiencias en el uso de la mano de obra. Estas se midieron por medio de toma de datos, análisis de métodos y tiempos, análisis estadístico de procesos y por medio de herramientas de calidad (diagramas de causa y efecto).
Las principales causantes de los reprocesos en la sección de pinturas en Muebles Bovel son: diseño de infraestructura inadecuada para un proceso productivo por piezas, herramientas no estandarizadas usadas en el transporte y desplazamiento de piezas, manipulación y almacenamiento temporal inapropiadas y falta de control sobre el desempeño de las actividades desarrolladas.

Se puede determinar que las principales causas a eliminar o mitigar, son las identificadas como movimientos o técnicas.

Después de analizar los costos unitarios de materia prima y los costos unitarios de mano de obra para diferentes órdenes de producción de multimuebles, se puede observar que en los costos de materia prima las variaciones son pequeñas, pero en la mano de obra se encuentran diferencias significativas entre una orden de producción y otra.

Se estableció que la empresa tiene una rentabilidad aproximada promedio del $27,44 \%$ con el proceso actual y sus deficiencias logísticas, con lo cual se puede estimar que los costos de la no calidad en la producción, son de aproximadamente un promedio de $\$ 124.664$ por grupo de piezas.

Se planteó una propuesta bajo la cual se presenta una solución a un problema logístico, el cual se inicia desde el tipo de material, el diseño de las piezas, los sistemas de sujeción, el método de traslado, las áreas para almacenamiento y esperas, las personas involucradas en las actividades y la experticia de cada operario. Esta consiste en un carro para el traslado de piezas lijadas en blanco, sujetador de piezas, un halador para el desplazamiento por parte del operario, un sistema para la aplicación del sellador catalizado, sistema de secado de piezas con sellador catalizado, un sistema de rieles para el desplazamiento de las piezas en la cabina de pintura, un sistema de riel extendido hasta la cabina de secado, acondicionamiento del almacenamiento temporal y la cabina de inspección.

En el análisis de las actividades, con la propuesta se podría disminuir el total de actividades de 41 a 29; los transportes se reducen a la mitad y al contar con ayudas mecánicas se espera también la reducción en los tiempos, en las operaciones de pintura para múltiples caras tanto en sellador catalizado como en laca catalizada.

\section{RECOMENDACIONES}

Para lograr los resulados esperados, objeto del presente estudio, la empresa Muebles Bovel Ltda., debe hacer seguimiento a los sistemas diseñados y de este modo aprovechar mejor las virtudes de la propuesta. Adicionalmente es fundamental establecer una metodología para determinar los Costos Indirectos de Fabricación necesarios para realizar una orden de producción en Muebles Bovel Ltda. 


\section{REFERENCIAS}

[1] Bello Perez, Carlos. Manual de producción, aplicado a las pymes. Ed. EOE. Segunda Edición. Bogotá D.C., Agosto 2006. p 38.

[2] Bowersox, Donald J. y CLOSS, David J. Logistical Management: The Integrated Supply Chain Process. Ed. McGraw-Hill, Nueva York, 1996, p. 25.

[3] Harrington, H. James. Mejoramiento de los procesos de la empresa. Ed. Mc Graw Hill. Cali-Colombia, Abril.2000

[4] Long, Douglas. Logística Internacional, Administración de la cadena de abastecimiento global. Ed. Limusa S.A. México, D.F. ISBN-13: 978-968-18-6581-8

[5] Presencia, José. Calidad Total y Logística. Ed. LogisBook. Segunda Edición. Barcelona España, 2004.

[6] Koontz, Harold y Weihrich, Heinz. Elementos de Administración. Ed. Mc Graw Hill.

[7] Muñoz Z. Rubén Darío y MORA G. Luis Aníbal. Diccionario de logística y negocios internacionales. Tercera Edición. Ecoe Ediciones. Bogotá D.C., 2009. ISBN 978-958-648-605-7.

[8] Olve, Nils - Göran; ROY, Jan y WETTER, Magnus. Implantando y gestionando el cuadro de mando integral (performance drivers). Ediciones Gestión 2000 S.A., Barcelona, 2002.

[9] Orozco, Fernando R. Control de Calidad. Ed. Universidad Tecnológica de Pereira.Tercera Edición. Pereira Rda., 1989.

[10] Ospina garces, William. Proceso Administrativo Contemporáneo. Universidad Tecnológica de Pereira. Pereira, 2002.

[11] Abrigo, Juan Martin. Puentes Grúas. Sigweb, división difusión y comunicaciones. http://www.sigweb.cl/biblioteca/PuentesGruas.pdf

[12] Bahamon L.. José Hernando. Construcción de Indicadores de Gestión Bajo el Enfoque de Sistemas. S \& T. Revista de la Ingeniería de Sistemas e Ingeniería Telemática. Universidad ICESI. http://www.icesi.edu.co/es/publicaciones/publicaciones/con tenidos/ sistemas_telematica/1/jbahamon_const-indgestion.pdf

[13] Benitez Lopez, Erick Oscar Gestión de outsourcing Logístico para almacén de productos farmacéuticos. Tesis digitales
http://sisbib.unmsm.edu.pe/bibvirtualdata/Tesis/Ingenie/benites_ le/cap5.pdf

[14] Blue Hous Group. BH Industrie, Servicios Industrie. http://www.blue-haus.com/Servicios/industrie.aspx

[15] CENLIT. La Madera. Ed. Cenlit. http://www.cenlit.com/muestra.pdf..

[16] Arias Acevedo, Diana y García Osorio, Jorge. Planteamiento de un modelo logístico para reducir costos del subproceso de pintura en muebles Bovel ltda. Universidad Tecnológica de Pereira, 2011. 